\title{
Measurement Techniques for Electromagnetic Shielding Behavior of Braided-Shield Power Cables: An Overview and Comparative Study
}

\author{
Peng Hu, Zhongyuan Zhou, Jinpeng Li, Xiang Zhou, Mingjie Sheng, Peng Li, Qi Zhou \\ Electromagnetic Compatibility Laboratory, School of Mechanical Engineering, Southeast University, Nanjing 211189, China, \\ zyzhou@seu.edu.cn
}

\begin{abstract}
More and more EMC tests have shown that the radiated emission problems of the equipment under test mainly concentrate on the interconnected power cables and cable connectors. Measurement of shielding performance is a prerequisite for quantitative and qualitative evaluation of the frequency-dependent characteristic of braided-shield power cables and cable connectors. Due to the asymmetric geometric structures of these cable assemblies, compared with the coaxial and symmetrical communication cables, the commonly used transfer impedance testing methods may not be suitable. In view of this, several improved simple and effective measurement methods, including transfer impedance and shield reduction factor testing methods, were proposed in recent years. These methods, based on the equivalent circuit model of the characteristic parameters, provide good repeatability for the measurement of shielding performance. This paper presents an overview analysis of various measurement techniques for shielding performance of power cables and cable connectors, highlights some of its equivalence principle in measurement setups, and showcases a brief comparison between transfer impedance and shield reduction factor.
\end{abstract}

Keywords: Braided-shield power cable, shielding performance, measurement techniques, transfer impedance, shield reduction factor.

\section{INTRODUCTION}

Braided-shield power cable is a common component widely used for the interconnection of various electrical devices. Relatively independent high-voltage high-power drive system, whether high-speed railway traction, electric ship propulsion, or electric vehicle drive system, will inevitably produce electromagnetic interference (EMI) due to the extensive application of unbalanced non-linear loads and highpower electronics. Moreover, the ever-increasing number of electromagnetic compatibility (EMC) tests indicate that the violation of radiated emission (RE) threshold limits of MILSTD-461G, European Norm EN55022/032 or other relevant standards mainly concentrates on the interconnected cables, so that a formerly quite neglected EMC problems is now widely investigated [1]-[3].

In general, the objective of braided-shield cable is to reduce significantly the effects of incident fields on sensitive circuits as well as to prevent the emission of components of the system from radiating outside the boundaries limited by the shield. The aspect ratio of braid shield is very often potentially responsible for an antenna-like behavior, and thus of the resulting emission and susceptibility problems in many installations [4]. For the selection of braided-shield power cable, especially considering the electromagnetic shielding requirements of practical engineering, mainly relies on characteristic parameters used to represent this specific coupling. Accordingly, there is no doubt that qualitative and quantitative eval- uation of electromagnetic shielding characteristic of cables should be carried out in accordance with the susceptibility and emission mechanism of transient electromagnetic fields (EMFs) to braid shield.

In view of the standards and existing literature, either parameter of transfer impedance and shield reduction factor is sufficient to fully describe the electromagnetic shielding behavior of cables [4]-[6]. The analytical methods (e.g., Vance [7], Tyni [8], Demoulin [9], Sali [10], and Kley [11] model, especially these models are discussed in [12]), and numerical methods (e.g., finite element method (FEM) [13], [14]) can provide frequency-dependent characteristic parameters of braid shield, respectively. Whereas these methods are useful to optimize the material and geometric parameters of braid shield, only direct measurement technique is feasible for the reliable qualitative and quantitative evaluation. It is worth noting, however, that EMC standards, e.g., IEC 62153-4-3 [15] and IEC 62153-4-6 [16], determine the testing methods of transfer impedance for coaxial and symmetrical communication cables only. Generally, power cables could have asymmetric geometric structures which might make the standard methods unapplicable as they are. For this reason, several investigations on the measurement of shielding performance for braided-shield power cables and cable connectors have been conducted in comparison with standard methods.

In this paper, a short overview on some main results and recent progress on measurement setups for shielding perfor- 
mance of power cables and cable connectors is presented. The paper is organized as follows. Investigation begins in Section 2 with a brief review of characteristic parameters for the qualitative evaluation of braid shield. Quantitative configurations in the frequency range from $25 \mathrm{~Hz}$ to $110 \mathrm{MHz}$ are discussed in Section 3, which can not only estimate the suppression effect of higher harmonics, but also provide effective estimates for the potential coupling of FM radio signals to cables. Section 4 presents a comparative analysis for transfer impedance and the shield reduction factor. The evaluation of the testing methods and the applicability of these methods are explained in Section 5. Finally, a summary is given in section 6.

\section{CHARACTERISTIC PARAMETERS}

\subsection{Transfer impedance}

The use of transfer impedance $Z_{t}$, as defined in Fig. 1, is well accepted for evaluating the electromagnetic shielding performance of braided-shield cables. The external timevarying EMFs coupling to inner conductor, i.e., corresponding to the effects of voltage source $V_{s s}$ and current source $I_{s s}$ on the equivalent circuit, will generate induced voltage $V_{i}$ and induced current $I_{i}$ in inner conductor, $V_{s}$ and $I_{s}$ in braid shield, respectively. In this way, for transfer impedance $Z_{t}$, these parameters are related to each other by equivalent voltage source $V_{s i}$ as [5], [12]

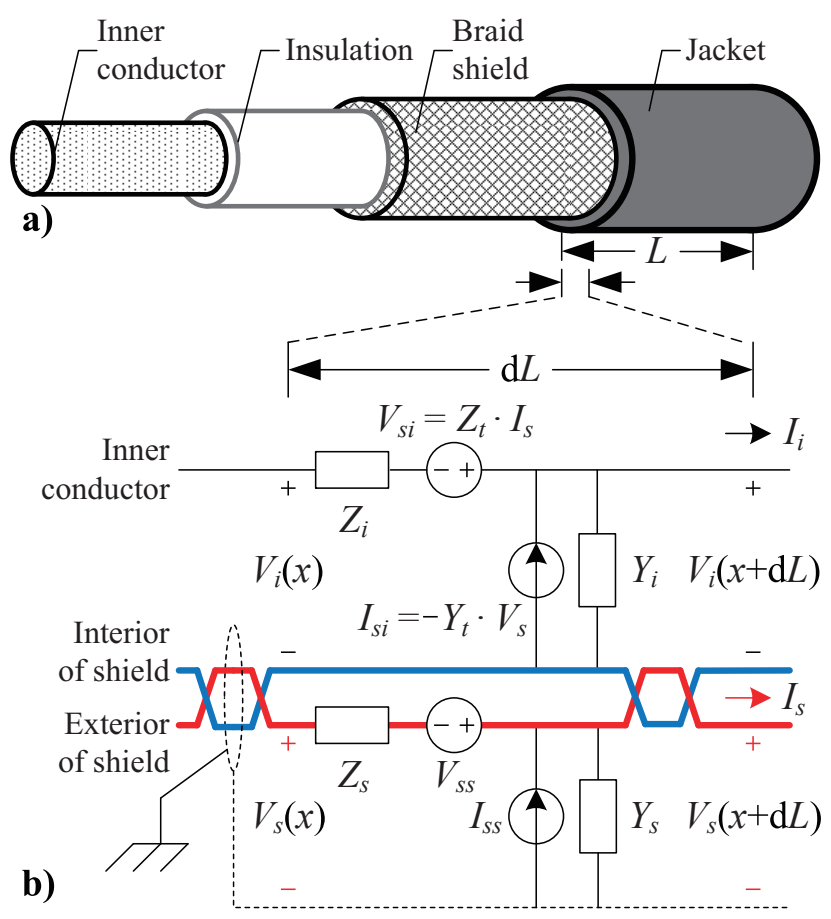

Fig.1. Basic structure a) of braided-shield cables, and equivalent circuit b) for an electrically short cable with an infinitesimal length $\mathrm{d} L$, i.e., equivalent circuits are formed between inner conductor and interior of braid shield, exterior of braid shield and ground. In specific, $Z_{i}\left(Y_{i}\right)$ and $Z_{S}\left(Y_{s}\right)$ represents equivalent impedance (admittance) per unit length for inner conductor and braid shield, respectively.

$$
\left\{\begin{array}{l}
\frac{\mathrm{d} V_{i}}{\mathrm{~d} L}+Z_{i} \cdot I_{i}=V_{s i} \\
V_{s i}=Z_{t} \cdot I_{s}
\end{array}\right.
$$

Then, transfer impedance $Z_{t}$ can be linearly derived from (1) in terms of $I_{i}=0$, and defined as the ratio of induced conductor-to-shield voltage per unit length to the shield current:

$$
Z_{t}(\Omega / \mathrm{m})=\left.\frac{1}{I_{S}} \cdot \frac{\mathrm{d} V_{i}}{\mathrm{~d} L}\right|_{I_{i}=0}
$$

\subsection{Shield reduction factor}

In [5], the concept of shield reduction factor, $K_{r}$, provides an unambiguous evaluation criterion for the effectiveness of braided-shield cables, and thus, constitutes another particularly convenient means of the shielding performance. The equivalent circuit similar to Fig. 1, shows the induced voltage $V_{i g}$ and $V_{i s g}$ in Fig. 2 as functions of $I_{i g}, I_{s}$ and impedance parameters. The shield reduction factor $K_{r}$ in $\mathrm{dB}$, can basically be defined as the ratio of induced voltage $V_{i s g}$ appearing, inner conductor to shield at the receiving end of the cable, to induced voltage $V_{i g}$ applied in series into the loop without braid shield [5], [17], viz.,

$$
K_{r}(\mathrm{~dB})=20 \lg \left(\frac{V_{i s g}}{V_{i g}}\right)
$$

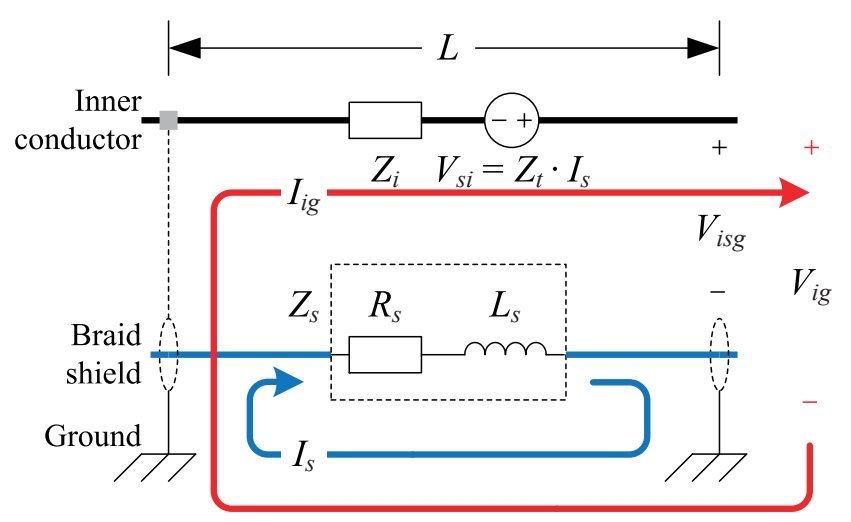

Fig. 2. Conceptual view of shield reduction factor. Red arrow line: induced conductor-to-ground current $I_{i g}$ without braid shield, and the corresponding voltage represented as $V_{i g}$. Blue arrow line: induced shield to ground current $I_{S}$ with braid shield, and the corresponding conductor to braid shield voltage denoted as $V_{i s g}$.

With the shield grounded induced voltage $V_{i s g}$ mainly depending on $Z_{t}$. Note that both ends of the shield are considered to be connected to an ideal ground, and consequently $V_{i s g}=V_{s i}$. On the contrary, if there is no shield or shield with floating ground, induced voltage $V_{i g}$ is positively correlated with impedance (denoted as $Z_{\text {loop }}$ ) of the inner conductor to 
ground loop, and $V_{i g}=I_{i g} \cdot Z_{\text {loop }}$. Therefore, shield reduction factor $K_{r}$ can be rewritten as

$$
K_{r}(\mathrm{~dB})=20 \lg \left(\frac{I_{s} \cdot Z_{t}}{I_{\text {ig }} \cdot Z_{\text {loop }}}\right)
$$

\subsection{Other characteristic parameters}

In addition to the characteristic parameters previously mentioned, screening attenuation $a_{s}$ and shielding effectiveness (SE) can also be used to estimate the performance of braid shield. Screening attenuation, $a_{s}$, usually expressed in $\mathrm{dB}$, is defined as the ratio of feeding power $P_{g}$ to the radiated maximum power $P_{\text {rad,max }}$ in the circuit [18]. And it can be expressed by

$$
a_{s}(\mathrm{~dB})=10 \lg \left(\frac{P_{g}}{P_{\text {rad }, \text { max }}}\right)
$$

It is worth noting at the outset that shielding performance of connectors should be no worse than that needed for cables under test to which they are attached. And screening attenuation $a_{s}$ can establish a relationship with transfer impedance $Z_{t}$ if one considers external circuit parameters over the measurement setup. However, screening attenuation is mainly used to measure the shielding performance of electrically long cables, and the lower the frequency, the longer the cable under test should be.

Similarly, SE of any configuration is defined as a ratio between two suitable electromagnetic power, electric field, or magnetic field values, namely

$$
\mathrm{SE}(\mathrm{dB})=20 \lg \left(\frac{E(\text { or } H) \text { without shield }}{E(\text { or } H) \text { with shield }}\right)
$$

As per [4] SE is a measure of the reduction or attenuation of the EMFs at a given point in space caused by the insertion of a shield between the source and that point. Obviously, SE as well as screening attenuation $a_{s}$ can provide an intuitively complete analysis of attenuation of electromagnetic energy to braid shield. Nevertheless, there is still some difficulty due to the actual dimensions of cables, which make the insertion of field probes inside the shield difficult. And the measurement suffers from several drawbacks: the two setups (with and without shield) will differ in their terminal conditions because of the influence of braid shield on the line characteristics, namely the propagation constant and the characteristic impedance. In addition, mechanism of electromagnetic radiation for cable with and without shield is significantly different. In view of these, SE is not suitable, on the one hand, the two configurations must be identical, and the requirement is not easy to achieve [4], on the other hand, the mechanism of electromagnetic radiation of cable should be considered.

\section{Measurement TeCHNiQues}

\subsection{Triaxial method}

Triaxial method (TM), a measurement setup as shown in Fig. 3, is a classical method for the transfer impedance measurement of coaxial and symmetrical cables (electrically short) as well as screening attenuation (electrically long). TM used for transfer impedance measurement, in general, is suitable in the frequency range $10 \mathrm{kHz}$ to a cut-off frequency $f_{\text {tri,cut }}$ which mainly depends on the coupling length $L$ discussed in detail in [15]. The so-called triaxial structure is consists of inner conductor, braid shield and tube, where the equivalent circuit of the former two is regarded as the primary loop, and the latter two as the secondary loop. Contrary to the equivalent voltage source $V_{s i}$ shown in Fig. 1, the equivalent voltage source $V_{2}$ related to transfer impedance $Z_{t}$ should exist in the secondary loop in case of reciprocity. Obviously, transfer impedance $Z_{t}$ shown in Fig. 3.b) can be derived according to

$$
Z_{t}(\Omega / \mathrm{m})=\frac{1}{L} \cdot \frac{V_{2}}{I_{s}}
$$

It is worth remarking that $R_{1}$ in the primary loop, should be matched with the characteristic impedance $Z_{p r i}$ of the cable under test, while $R_{2}$ does not have to be equal to the characteristic impedance $Z_{s e c}$ of the secondary loop. To simplify the analysis procedure, we make an assumption that $R_{1}=Z_{p r i}$. Otherwise, for the case where reflection coefficient can not be ignored, the relevant detailed explanation is discussed in [19].

Considering the equivalent circuit of secondary loop in the measurement setup, $V_{2}$ can be derived from $V_{r}$, i.e., directly measured by receiver. Referring to the equivalent circuit of primary loop, $I_{S}$ can be easily calculated. Note that the

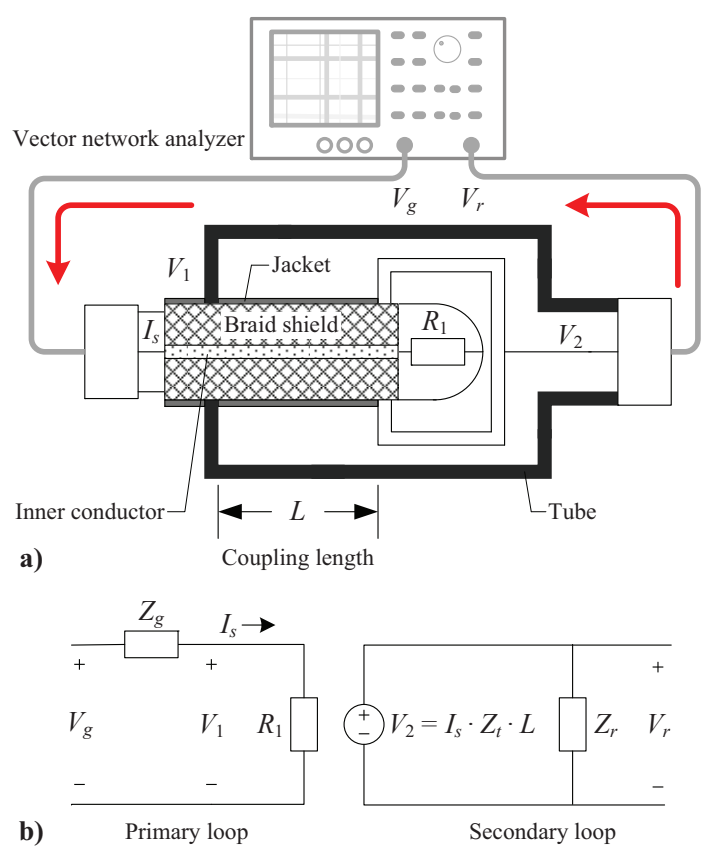

Fig. 3. Measurement setup a) with vector network analyzer, and equivalent circuit $b$ ) for the triaxial method. The impedance of the generator and receiver are $Z_{g}$ and $Z_{r}$, respectively, and in general $Z_{g}=Z_{r}=50 \Omega$. 
conductor-to-shield voltage $V_{1}$ is provided by a signal generator (source voltage denoted as $V_{g}$ ) instead of excitation over electromagnetic induction. Thus, for the primary loop, $I_{s}=V_{1} / R_{1}$, and (7) is rewritten as

$$
Z_{t}(\Omega / \mathrm{m})=\frac{R}{L} \cdot \frac{V_{r}}{V_{1}}
$$

Specially, for a calibrated receiver or vector network analyzer (VNA), the second term on the right side of (8) is

$$
\frac{V_{r}}{V_{1}}=10^{-a_{\text {meas }} / 20}
$$

Where $a_{\text {meas }}$ is the attenuation during the measurement operation, and defined by taking account of the power $P_{g}$ fed to the primary loop and the power $P_{r}$ fed back to the receiver over secondary loop, or of the fact that the scattering transmission parameter $S_{21}$ are measured by VNA, hence

$$
a_{\text {meas }}=10 \lg \left(\frac{P_{g}}{P_{r}}\right)=-20 \lg \left(S_{21}\right)
$$

If the coupling length $L$ is electrically long, TM shown in Fig. 3 can be used for the measurement of screening attenuation $a_{s}$, and (5) can be written as [20]

$$
a_{S}(\mathrm{~dB})=10 \lg \left(\frac{P_{g}}{P_{\text {rad }, \max }}\right)=10 \lg \left|\frac{2 Z_{N}}{Z_{r}} \cdot \frac{P_{g}}{P_{r, \text { max }}}\right|
$$

Where $Z_{N}$ is the normalized value of the characteristic impedance of the environment of a typical cable installation, and $P_{r, \text { max }}$ is the measured power received on the input impedance $Z_{r}$ in the secondary loop. In addition, $P_{r, \max }$ and $P_{g}$ can be related to the transfer impedance $Z_{t}$ at high frequencies according to [20]

$$
\sqrt{\frac{P_{r, \text { max }}}{P_{g}}} \approx \frac{c}{\omega \sqrt{R_{1} Z_{r}}} \cdot\left|\frac{Z_{t}+Z_{f}}{\sqrt{\varepsilon_{r 1}}+\sqrt{\varepsilon_{r 2}}}+\frac{Z_{t}-Z_{f}}{\sqrt{\varepsilon_{r 1}}-\sqrt{\varepsilon_{r 2}}}\right|
$$

Where $c$ is vacuum velocity, $Z_{f}$ is the capacitive coupling impedance of the cable under test, $\varepsilon_{r 1}$ and $\varepsilon_{r 2}$ represent relative dielectric permittivity of the cable under test and the secondary loop respectively.

\subsection{Line injection method}

Line injection method (LIM) is another classical method for the transfer impedance measurement of coaxial and symmetrical cables in the frequency range from a few $\mathrm{kHz}$ up to and above $1 \mathrm{GHz}$ [16], the basic configuration with VNA is shown in Fig. 4.a). If the output signal of VNA is provided as shown by the red arrow, and the coupling length $L \ll \lambda_{\text {line,cut }}$ (wavelength corresponding to the cut-off frequency), the equivalent circuit for LIM is shown in Fig. 4.b), where electrical connections form the primary loop and secondary loop over shield-to-injection line and inner conductorto-braid shield, respectively.

It is worth noting, again, that the prerequisite for the equivalent electrical model is that the cable under test should be electrically short, and $R_{1}$ and $R_{2}$ (shown in Fig. 4) are well matched to the characteristic impedance of the primary loop, and secondary loop respectively. As per [19], the coupling length $L$, should not exceed one-tenth of the $\lambda_{\text {line,cut }}$, to be considered as electrically short, e.g., $L=0.5 \mathrm{~m}$, and the upper frequency is only $60 \mathrm{MHz}$. In view of this, the transfer impedance $Z_{t}$, as shown in Fig. 4.b), can also be derived in terms of (7),

$$
Z_{t}(\Omega / \mathrm{m})=\frac{1}{L} \cdot \frac{R_{1}\left(R_{2}+Z_{r}\right)}{Z_{r}} \cdot \frac{V_{r}}{V_{1}}
$$

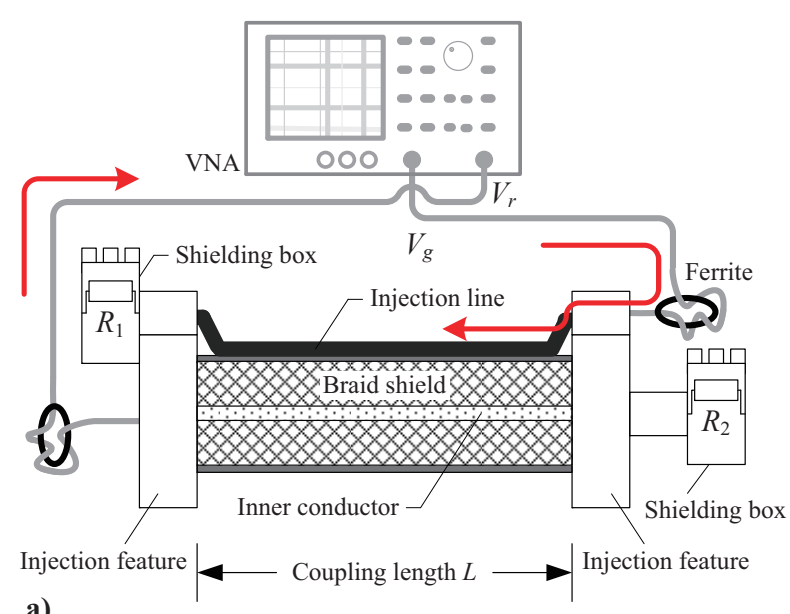

a)

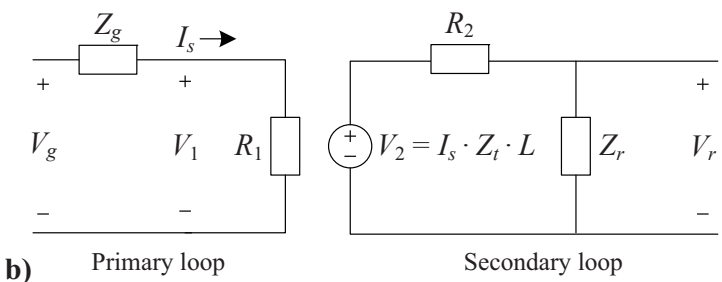

Fig. 4. Measurement setup a) with vector network analyzer, and equivalent circuit b) for the line injection method. Ferrites are used to aviod ground-loop effects.

\subsection{Current probe method}

Current probe method (CPM) is a relatively simple way to obtain transfer impedance $Z_{t}$ of the cable under test [21], [22]. The measurement configuration is illustrated in Fig. 5. During the measurement operation, electric signal is injected into the braid shield-to-ground loop (i.e., the primary loop) over the injection probe, and consequently, the equivalent voltage source $V_{2}$ related to $Z_{t}$ can be regarded as the main power source in the shield-to-inner conductor loop (i.e., the secondary loop) that both sides are well matched with terminal impedance $R_{1}$. On the one hand, the receive port of the VNA connects with the monitor probe, the shield-to-ground current $I_{S}$ is expressed by the scattering transmission parameter $S_{21, m}$ (i.e., the red arrow shown in Fig. 5.b)), while on 
the other hand the receive port, instead of a well-matched impedance $R_{1}$ adjacent to the monitor probe, directly connects with the secondary loop, and scattering parameter $S_{21, l}$ related to the power of $V_{2}$ is measured (i.e., the blue arrow).

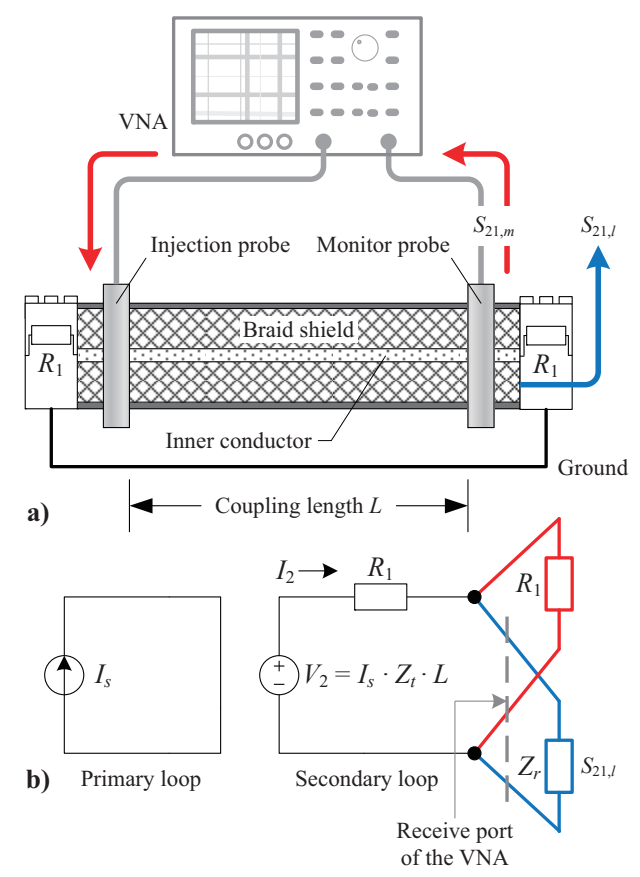

Fig. 5. Measurement setup a) with VNA, and equivalent circuit b) for the current probe method.

Suppose that the coupling length of the cable under test is electrically short, and the secondary loop is well matched (the characteristic impedance $Z_{s e c}$ and $R_{1}$ should be equal to the port impedance $Z_{r}$ of VNA, and in general, $Z_{\text {sec }}=R_{1}=$ $Z_{r}=50 \Omega$ ). As we all know, the scattering parameter has the dimensions of the square root of power, $S_{21, m}$ and $S_{21, l}$ can be written as

$$
S_{21, m}=\sqrt{\frac{P_{r, m}}{P_{g}}}, \quad S_{21, l}=\sqrt{\frac{P_{r, l}}{P_{g}}}
$$

Where $P_{g}$ is the power fed to the primary loop over the injection probe, $P_{r, m}$ and $P_{r, l}$ represent the power fed back to the receive port, respectively. Referring to Fig. $5, P_{r, m}$ is

$$
P_{r, m}=\frac{V_{r, m}^{2}}{Z_{r}}=\frac{\left(I_{s} \cdot Z_{t t}\right)^{2}}{Z_{r}}
$$

and $P_{r, l}$ is

$$
P_{r, l}=I_{2}^{2} \cdot Z_{r}
$$

Where $V_{r, m}$ is terminal voltage of the receive port, $Z_{t t}$ represents the transfer impedance between the monitor probe and the shield, $I_{2}$ is the current in secondary loop. Obviously, $I_{S}$ can be easily derived by combining (14) and (15),

$$
I_{s}=\frac{\sqrt{P_{g} \cdot Z_{r}}}{Z_{t t}} \cdot S_{21, m}
$$

and $I_{2}$ is

$$
I_{2}=\frac{U_{2}}{2 R_{1}}=\frac{I_{s} \cdot Z_{t} \cdot L}{2 R_{1}}=\frac{Z_{t} \cdot L \cdot S_{21, m}}{2 Z_{t t}} \cdot \sqrt{\frac{P_{g}}{Z_{r}}}
$$

Then, if we substitute (14) and (16) into (18) we obtain the following relationship:

$$
Z_{t}(\Omega / \mathrm{m})=\frac{2 Z_{t t} \cdot S_{21, l}}{L \cdot S_{21, m}}
$$

\subsection{Ground plate method}

Ground plate method (GPM) is improved by the commonly used TM, and especially designed for braided-shield power cables and cable-connector assemblies [23]-[25]. The configuration is shown in Fig. 6. A copper plate is used in the secondary loop instead of tube structure as shown in Fig. 3.a), and it is apparent that the equivalent circuit for conductor-toshield and shield-to-copper plate can be regarded as primary loop and secondary loop, respectively. If the output signal of VNA is provided as shown by the blue arrow, copper plate is used in the primary loop instead of the injection line, and consequently the equivalent circuit shown in Fig. 4.b) is also suitable for GPM.

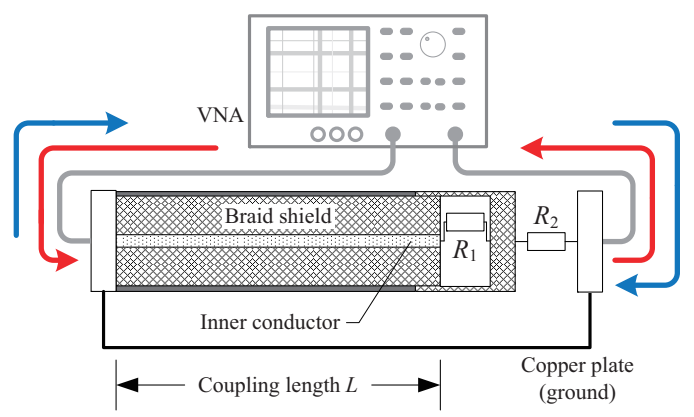

Fig.6. Measurement setup for the ground plate method.

\subsection{Shield reduction factor method}

Shield reduction factor method (SRFM) is an effective way to evaluate the frequency-dependent shielding performance from a few $\mathrm{Hz}$ to a few MHz. According to the definition of shield reduction factor $K_{r}$ in (3), the measurement setup is described in detail in Fig. 7.a). When current is injected into the shield (see black arrow dashed line), the single-pole double-throw (SPDT) switch $S$ connects to terminal $A$ and $B$, and the corresponding induced voltages $V_{A}$ and $V_{B}$ are measured, respectively. Then, it is clear that the ratio of $V_{A}$ to $V_{B}$ can be understood as $V_{i s g} / V_{i g}$ in (3). Regarding electromagnetic susceptibility or emission of the cable under test, the induced shield current $I_{s}$ is considered as having a gradually decreasing current density $J_{S}$ as shown in Fig. 7.a). In [12], these variations can be approximately determined by the skin depth of the material of braid shield. Yet, skin depth influence is rapidly overcast by the leakages of the braid weaving. 
In other words, the skin depth also indirectly determines the upper frequency limit of SRFM. However, it is worth noting that the source is directly loaded onto the braid shield, it is uncertain how many amperes will have to be supplied by the power source to ensure that the measurement for induction voltage has a sufficient dynamic range [17], [26].

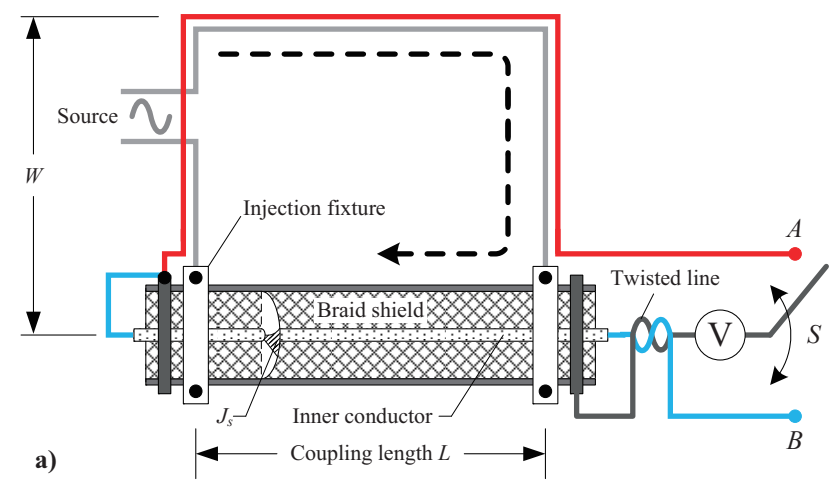

b)

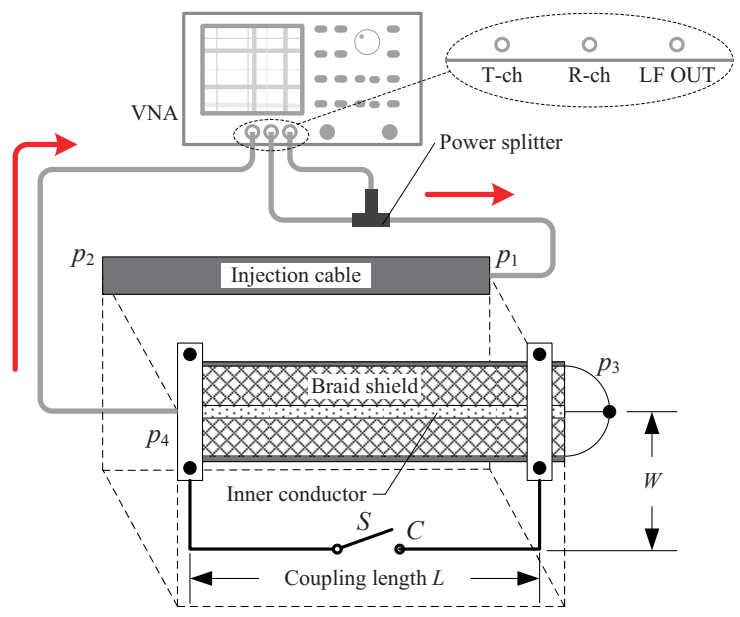

Fig. 7. Measurement setup for the shield reduction factor method.

In [26], an improved SRFM was proposed, and the gainphase test port $(5 \mathrm{~Hz}-30 \mathrm{MHz})$ of Agilent E5061B VNA was used not only as a broadband power supply but also as a highprecision receiver with a dynamic range of greater than 90 dB. The configuration is shown in Fig. 7.b), the cable under test and injection cable are arranged in parallel with the same height, where one part of the output of low frequency (LF) source is injected into $p_{1}$ with a power splitter, the other part is fed back to the R-ch receiver of VNA, and the induced electrical signal of $p_{4}$ is measured by T-ch receiver. For terminals $p_{2}$ and $p_{3}$, the inner conductor directly connects to braid shield. When the switch $S$ connects to terminal $C$, a $L \times W$ rectangular loop is formed, which means that both sides of braid shield are well grounded, and effectively suppress the induced voltage (i.e., $V_{\text {isg }}$ in (3)) between inner conductor and ground, and the corresponding measurement parameter of VNA is denoted as $T_{1} / R$. Conversely, the $L \times W$ rectangular loop is open, and the measured parameter of VNA, denoted as $T_{2} / R$, is mainly dependent on the induced voltage (i.e., $V_{i g}$ in (3)). Note that
$R$ measured by $\mathrm{R}$-ch receiver over two measurements can be considered as a constant value, and consequently, $K_{r}$ is rewritten as

$$
K_{r}(\mathrm{~dB})=20 \lg \left(\frac{T_{1}}{R}\right)-20 \lg \left(\frac{T_{2}}{R}\right)=20 \lg \left(\frac{T_{1}}{T_{2}}\right)
$$

Referring to Fig. 7, the $L \times W$ rectangular loop is closely related to the value of $K_{r}$, regardless of the method used for measurement. In fact, the impedance of $L \times W$ rectangular loop is $Z_{\text {loop }}$ in (4), i.e., when the coupling length $L$ is determined, $W$ can be easily calculated by $Z_{\text {loop }}$, and the relevant formulas are already pointed out in [26]-[28].

\section{DISCUSSION AND ANALYSIS}

Measurement of SRFM was carried out as arranged similarly to Fig. 7.b) in the frequency range $25 \mathrm{~Hz}$ to $1 \mathrm{MHz}$. Fig. 8 shows the test value $K_{r}$, the derivation $Z_{t}$ of $K_{r}$, obviously, these values unveil three basic frequency ranges, i.e., domain I, II, and III.
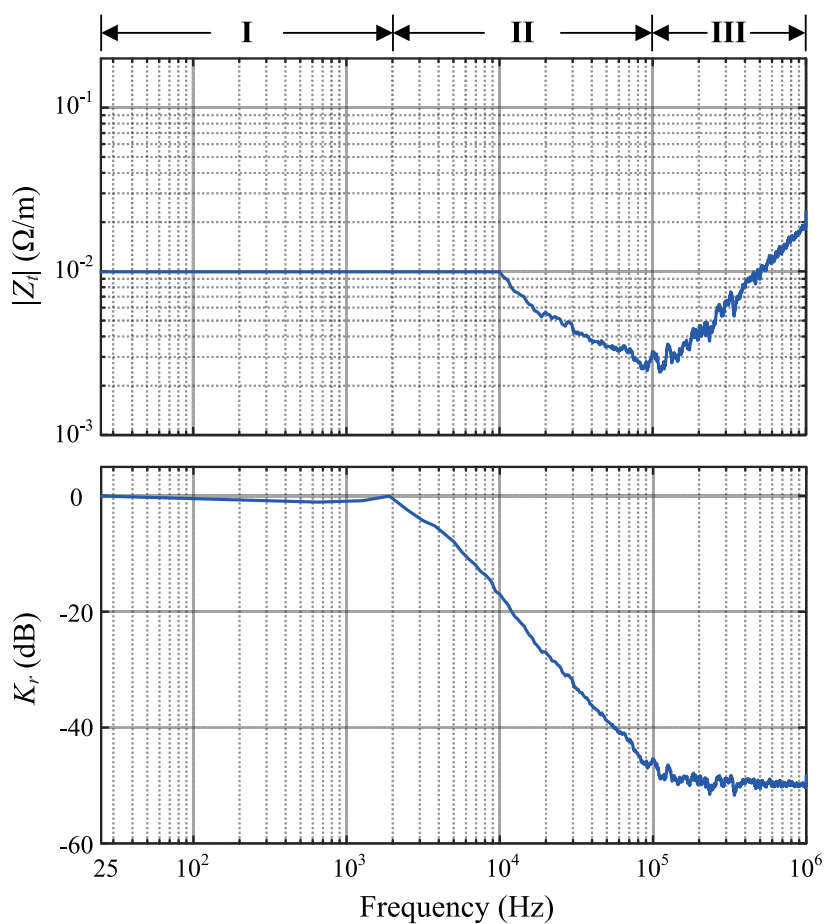

Fig. 8. Frequency-dependent magnitude of transfer impedance $Z_{t}$ (Upper) and shield reduction factor $K_{r}$ (Lower). $Z_{t}$ is the derivation calculated by the test value of $K_{r}$ from $25 \mathrm{~Hz}$ to $1 \mathrm{MHz}$, the braidedshield power cable under test is CJPF 96 / SC $2 \times 25 \mathrm{~mm}^{2}$ provided by Changzhou Marine Cable Co., Ltd. (China).

As previously mentioned in (4), the frequency-dependent characteristic of $K_{r}$ mainly relies on $Z_{t}$ and $Z_{\text {loop }}$. Without loss of generality, we can assume that $Z_{t}=R_{t}+j \cdot \omega \cdot L_{t}$ and $Z_{\text {loop }}=$ $R_{s}+j \cdot \omega \cdot L_{\text {loop }}$, i.e., impedance can be regarded as series resistance and inductance. In general, another assumption in 
[17] is that $R_{t} \approx R_{s}$, and therefore, (4) is rewritten as

$$
K_{r}(\mathrm{~dB}) \approx 20 \lg \left(\frac{R_{s}+j \cdot \omega \cdot L_{t}}{R_{S}+j \cdot \omega \cdot L_{\text {loop }}}\right)
$$

More precisely, the value of $L_{t}$ is about several $\mathrm{nH} / \mathrm{m}$ for the common single layer braid, while $L_{\text {loop }}$ is about $1 \mu \mathrm{H} / \mathrm{m}$ at a height of 50 to $500 \mathrm{~mm}$ above ground [5], [29].

Regarding domain I, i.e., below $2 \mathrm{kHz}, R_{s} \gg \omega \cdot L_{t}$, and (21) can be simplified as [5], [17]

$$
K_{r}(\mathrm{~dB}) \approx 20 \lg \left(\frac{R_{S}}{R_{s}+j \cdot \omega \cdot L_{\text {loop }}}\right)
$$

Obviously, $K_{r}$ tends to a constant value approaching $0 \mathrm{~dB}$, and it means that the braid shield of the power cable under test offers relatively poor performance below $2 \mathrm{kHz}$. Note that the inductance of braid shield, $L_{S}$ (see Fig. 2), is included in $L_{\text {loop }}$, i.e., the inductance of $L \times W$ rectangular loop. Therefore, the value of $L_{S}$ can be increased appropriately in order to improve the shielding performance in the frequency range of domain I, i.e., conductive materials with relatively high permeability can be used for braid shield. Similar to the prerequisite of (22), $R_{S} \gg \omega \cdot L_{t}, Z_{t}$ dominated by $R_{S}$ is frequencyindependent, and $Z_{t} \approx 9.93 \mathrm{~m} \Omega / \mathrm{m}$.

Regarding domain II from $2 \mathrm{kHz}$ to $100 \mathrm{kHz}, Z_{\text {loop }}$ relies on $\omega \cdot L_{\text {loop }}$, the corresponding range is approximately from 0.01 to 0.6 , and (21) is

$$
K_{r}(\mathrm{~dB}) \approx 20 \lg \left(\frac{R_{s}+j \cdot \omega \cdot L_{t}}{j \cdot \omega \cdot L_{\text {loop }}}\right)
$$

$K_{r}$ is negatively correlated with frequency, and Fig. 8 also shows the test value of $K_{r}$ has similar frequency-dependent behavior, i.e., $K_{r}$ decreases with increasing frequency. Referring to $\omega \cdot L_{t}$, the maximum value is about $1.9 \times 10^{-3}\left(K_{r}=-50\right.$ $\mathrm{dB})$. Considering the equivalent model of series resistance $R_{S}$ and inductance $L_{t}, Z_{t}$ still depends on $R_{s}$, and $Z_{t}$ is negatively correlated with frequency from $10 \mathrm{kHz}$ to $100 \mathrm{kHz}$. As per [12] and [25], $Z_{t}$ decreases with frequency mainly relies on the scattering part (denoted as $Z_{d}$ ) of $R_{s}$, namely

$$
Z_{t} \approx Z_{d}=R_{s} \frac{m_{\delta}}{\sinh \left(m_{\delta}\right)}
$$

Where $m_{\delta}$ is positively correlated with $d / \delta$, and $d$ is the thickness of single braid wire, $\delta$ is the skin depth. As frequency increases, the skin depth $\delta$ decreases, and the corresponding $m_{\delta}$ increases. Specifically, if $m_{\delta} \leqslant 1$, then $\sinh \left(m_{\delta}\right) \approx m_{\delta}$, i.e., $f \leqslant 10 \mathrm{kHz}$, and $Z_{t} \approx R_{s}$. Otherwise, if $m_{\delta}>1$, then $\sinh \left(m_{\delta}\right)>m_{\delta}$, and consequently, $Z_{t}$ decreases with increasing frequency in terms of (24).

Regarding domain III above $100 \mathrm{kHz}$, the inductance component in $Z_{t}$ is gradually significant, and $Z_{t}$ increases linearly with the frequency. Therefore, (23) can also be further simplified as

$$
K_{r}(\mathrm{~dB}) \approx 20 \lg \left(\frac{L_{t}}{L_{\text {loop }}}\right)
$$

In view of this, $K_{r}$ keeps a constant value related to the material and geometric parameters of braid shield. Note that either parameter of $K_{r}$ and $Z_{t}$ is sufficient to describe the effect of suppression on common mode (CM) conduction coupling, not only for radiated coupling. In addition, as discussed in [5], the concept of $K_{r}$ indicates that it can be used for evaluating shielding performance of emission as well as susceptibility.

\section{EVAluATION AND SELECTION OF TEST METHODS}

Quantitative evaluation of electromagnetic shielding performance of braided-shield power cables in the frequency range $25 \mathrm{~Hz}$ to $110 \mathrm{MHz}$ cannot be achieved by only one configuration discussed in Section 3. Regarding frequency below a few $\mathrm{MHz}$, there is no doubt that SRFM is the most effective way to evaluate the frequency-dependent characteristic of power cables as well as cable connectors. As discussed in Section $4, K_{r}$ provides a more intuitive shielding effect analysis than transfer impedance $Z_{t}$ in ohmic region. At higher frequency (typically above $1 \mathrm{MHz}$ ), TM, LIM, CPM, and GPM can be used due to the resonance effect of SRFM.

Considering the difference of geometric characteristics (diameter of cable, shape of connector, etc.) of power cables and cable connectors (see Fig. 9.a)), different sizes of triaxial cubes and fixtures in TM should be integrated before each test session, which makes setup preparation time consuming and expensive [25].

LIM can be used for coaxial and symmetrical cables and cable connectors (e.g., type N, BNC and SMA connector), and test results in [30] demonstrate good repeatability. Note that the advantage of LIM, compared with TM, can delay the appearance of resonance caused by measurement configuration. Nevertheless, different results of transfer impedance $Z_{t}$ are given over different orientations of injection line along the sample under test (i.e., the asymmetric power cables and cable connectors) [23],[25]. Moreover, as per a test report provided by STIEE, the deviation of $Z_{t}$ results caused by the asymmetrical power cables and cable connectors is approximately $1 \mathrm{~m} \Omega / \mathrm{m}$ from $100 \mathrm{kHz}$ to $120 \mathrm{MHz}$ [31]. When the frequency is low, the corresponding value of $Z_{t}$ is about several $\mathrm{m} \Omega / \mathrm{m}$, the orientation of injection line along the sample has a significant influence on the test results of $Z_{t}$. In order to reduce the influence of deviation on test results, multiple comparison test for different orientations of injection line along the sample should be carried out for the same, and it means that LIM may take a long time.

CPM and GPM are barely independent of the geometric characteristics of power cables and cable connectors. Obviously, the typical cable-connector assembly in Fig. 9.b) can be regarded as cable under test discussed previously. It is worth noting, however, that the transfer impedance $Z_{t}(\Omega)$ of connector should remove the effects of power cables with coupling length $L-L_{\text {connec }}$ from the measurements. Referring to CPM, when the current probe is in different positions, i.e., different measurement locations of $I_{s}$, the test results are almost unaffected [32]. GPM provides a more flexible testing procedure of transfer impedance $Z_{t}$, and covers a widefrequency range from $10 \mathrm{kHz}$ to $300 \mathrm{MHz}$ over well-matched 


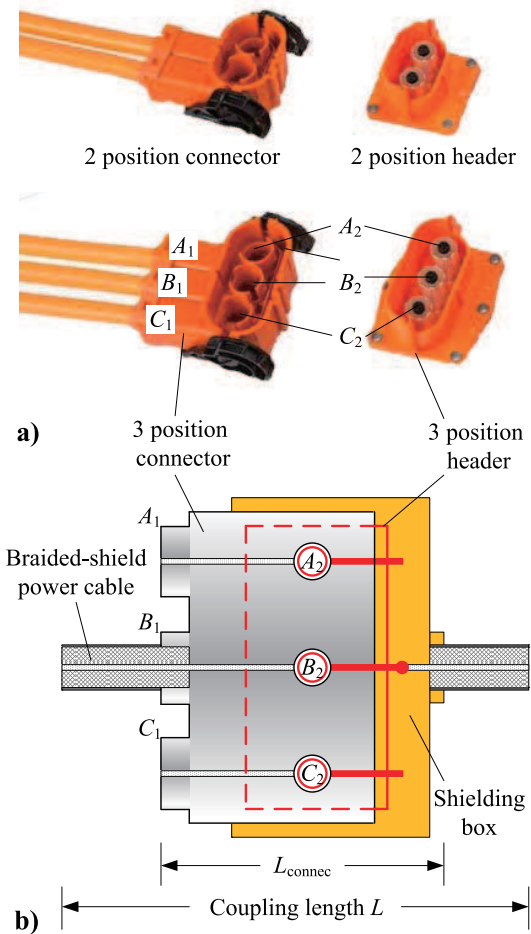

Fig. 9. Picture of the typical 2- and 3- position power cableconnector assemblies a), and the corresponding sample under test b). In specific, $A_{1}, B_{1}$, and $C_{1}$ represent the port between power cable and connector, respectively. The header is assembled on a shielding box, $A_{2}, B_{2}$, and $C_{2}$ are the inner conductors of connector and header. Arbitrary port, together with shielding box and the braided-shield power cables at both ends, are used to form a complete quasi-coaxial structure.

primary and sencondary loops [25]. Test results compared with TM and LIM show that GPM can be used as an alternative method for the commonly used measurement methods of transfer impedance $Z_{t}$. The obvious disadvantage is the real difficulty to weld the chip-size surface mounted resistors for well-matched impedances of primary and sencondary loops.

Consequently, for low frequencies (typically below 1 $\mathrm{MHz}$ ), SRFM can be used as an alternative to the transfer impedance methods, and provide more intuitive evaluation of shielding performance in ohmic region. As frequency increases to $110 \mathrm{MHz}, \mathrm{LIM}$ is the first choice for the $Z_{t}$ measurement of coaxial and symmetrical power cables and cable connectors. Regarding the asymmetric power cables and cable connectors, either CPM or GPM can replace the standard TM and LIM, and provide alternative measurement setup.

\section{CONClusions}

The first aim of this paper was to provide an overview of existing measurement methods for electromagnetic shielding performance of braided-shield power cables. To that end, characteristic parameters for the braid shield of cables, including transfer impedance and shield reduction factor, are discussed in terms of the equivalent circuit. And the corresponding transfer impedance and shield reduction factor methods are of different usable frequency ranges, different measurement configurations, suitable for different types of power cables and cable connectors, and have been utilized for different purposes in the past.

A brief comparative analysis for transfer impedance and shield reduction factor, reported in the paper, illustrated that shield reduction factor at low frequencies (typically below 1 $\mathrm{MHz}$ ) is more intuitive for evaluating the shielding performance of braid shield in ohmic region. For higher frequencies (1 MHz to $110 \mathrm{MHz}$ ), transfer impedance increases linearly with the frequency, and consequently, the corresponding transfer impedance methods are more suitable.

Among those, SRFM is the simple and more effective one at lower frequencies, regardless of the geometric characteristics of cables and cable connectors. For TM, the usable cable types are limited and it is not suitable for cable connectors, while LIM can be used for the measurement of coaxial and symmetrical samples. CPM and GPM are barely independent of the geometric structures of cables and cable connectors, and can be used as an alternative test procedure to TM and LIM.

\section{REFERENCES}

[1] Baklezos, A.T., Nikolopoulos, C.D., Katsouris, A.G., Koutantos, G.I., Capsalis, C.N. (2016). Electromagnetic emission modeling in case of shielded cabling with respect to the ground dielectric properties. IEEE Transactions on Electromagnetic Compatibility, 58 (6), 16941700.

[2] Yousaf, J., Amin, M., Iqbal, S. (2013). Investigation of low frequency emissions due to interfacing cables in RE 102. In International Conference on Space Science and Communication (IconSpace), Melaka, Malaysia: IEEE, 65-69.

[3] Du, Y.L., Lu, Y.H., Zhang, J.L. (2013). Eavesdropping the display image from conducted emission on network cable of a PC. The Journal of China Universities of Posts and Telecommunications, 20 (3), 78-84.

[4] Celozzi, S., Araneo, R., Lovat, G. (2008). Electromagnetic Shielding. John Wiley \& Sons, Inc., 200-209.

[5] Mardiguian, M. (2014). Controlling Radiated Emissions by Design, (3rd ed.). Springer, 291-301.

[6] International Electrotechnical Commission. (2014). Metallic communication cable test methods - Part 41: Electromagnetic compatibility (EMC) - Introduction to electromagnetic screening measurements. IEC TS 62153-4-1:2014. Geneva.

[7] Vance, E.F. (1975). Shielding effectiveness of braidedwire shields. IEEE Transactions on Electromagnetic Compatibility, EMC-17 (2), 71-77.

[8] Tyni, M. (1976). The transfer impedance of coaxial cables with braided outer conductor. In Digest of the 10th International Wroclaw Symposium on EMC, September 
1976. Wrclaw, Poland: Wissenschaftlicher Fachverlag Dr. Fleck, 410-419.

[9] Demoulin, B., Degauque, P., Cauterman, M. (1981). Shielding effectiveness of braids with high optical coverage. In Proceedings of the International Symposium on EMC. Zurich, Switzerland, 491-495.

[10] Sali, S. (1991). An improved model for the transfer impedance calculations of braided coaxial cables. IEEE Transactions on Electromagnetic Compatibility, 33 (2), 139-143.

[11] Kley, T. (1993). Optimized single-braided cable shields. IEEE Transactions on Electromagnetic Compatibility, 35 (1), 1-9.

[12] Tesche, F.M., Ianoz, M.V., Karlsson, T. (2009). EMC Analysis Methods and Computational Models, (Chinese ed.). Beijing, China: Beijing University of Posts and Telecommunications Press, 283-313.

[13] Otin, R., Verpoorte, J., Schippers, H. (2011). Finite element model for the computation of the transfer impedance of cable shields. IEEE Transactions on Electromagnetic Compatibility, 53 (4), 950-958.

[14] Otin, R., Verpoorte, J., Schippers, H., Isanta, R. (2015). A finite element tool for the electromagnetic analysis of braided cable shields. Computer Physics Communications, (191), 209-220.

[15] International Electrotechnical Commission. (2013). Metallic communication cable test methods - Part 4-3: Electromagnetic compatibility (EMC) - Surface transfer impedance - Triaxial method. IEC 62153-4-3:2013. Geneva.

[16] International Electrotechnical Commission. (2017). Metallic cables and other passive components test methods - Part 4-6: Electromagnetic compatibility (EMC) Surface transfer impedance - line injection method. IEC 62153-4-6:2017. Geneva.

[17] National Standards of the People's Republic of China. (2016). Test Methods for Communication Cable. GB/T 5441-2016. Beijing.

[18] International Electrotechnical Commission. (2006). Metallic communication cables test methods - Part 45: Electromagnetic compatibility (EMC) - Coupling or screening attenuation - Absorbing clamp method. IEC 62153-4-5:2006. Geneva.

[19] European Committee for Electrotechnical Standardization. (2002). Communication cables. Specifications for test methods. Electrical test methods. Electromagnetic performance. EN 50289-1-6:2002. Brussels.

[20] International Electrotechnical Commission. (2015). Metallic communication cable test methods - Part 44: Electromagnetic compatibility (EMC) - Test method for measuring of the screening attenuation $a_{s}$ up to and above $3 \mathrm{GHz}$, triaxial method. IEC 62153-4-4 (Edition 2.0):2015. Geneva.

[21] Morriello, A., Benson, T.M., Duffy, A.P., Cheng, C.F. (1998). Surface transfer impedance measurement: a comparison between current probe and pull-on braid methods for coaxial cables. IEEE Transactions on Electromagnetic Compatibility, 40 (1), 69-76.

[22] Benson, T.M., Duffy, A.P., Cheng, C.F. (1997). Assessing the performance of a current probe based method for determining surface transfer impedance. In 10th International Conference on Electromagnetic Compatibility, Coventry, UK: IET, 137-141.

[23] Mushtaq, A., Frei, S., Siebert, K., Barenfangeret, J. (2013). Analysis of shielding effectiveness of HV cable and connector systems used for electric vehicles. In International Conference on Electromagnetic Compatibility (EMC Europe), Brugge, Belgium: IEEE, 241-246.

[24] Mushtaq, A., Frei, S. (2016). Transfer impedance simulation and measurement methods to analyse shielding behaviour of $\mathrm{HV}$ cables used in Electric-Vehicles and Hybrid-Electric-Vehicles. Advances in Radio Science, (14), 139-145.

[25] Mushtaq, A., Frei, S. (2016). Alternate methods for transfer impedance measurements of shielded HVcables and HV-cable-connector systems for EV and HEV. International Journal of RF and Microwave Computer-Aided Engineering, 26 (4), 359-366.

[26] Zhou, Z.Y., Hu, P., Zhou, X., Li, J.P., Sheng, M.J., Li, P., Zhou, Q. (2019). Direct measurement of shield reduction factor of braided-shield cables in the frequency range of $25 \mathrm{~Hz}$ to $1 \mathrm{MHz}$. Nanjing: Southeast University.

[27] Theethayi, N., Thottappillil, R., Paolone, M., Nucci, C.A., Rachidi, F. (2007). External impedance and admittance of buried horizontal wires for transient studies using transmission line analysis. IEEE Transactions on Dielectrics and Electrical Insulation, 14 (3), 751-761.

[28] Rachidi, F., Tkachenko, S. (2008). Electromagnetic Field Interaction with Transmission Lines: From Classical Theory to HF Radiation Effects. WIT Press, 79-122.

[29] Yan, J.D. (1982). Computation of the Impact of Electric Wires on Communication Lines: Principles, Standards and Examples, (Chinese ed.). Beijing, China: Posts and Telecom Press, 219-220.

[30] Bluhm, M., Peroglio, E., Pierucci, G., Squizzato, V., Zich, R.E. (2000). Measurements of transfer impedance with the line injection method on cables and connectors. In International Symposium on Electromagnetic Compatibility, Washington, DC, USA: IEEE, 599-604.

[31] Typo Electronics (Shanghai) Co., Ltd. (2016). HVP 800/ HVA 280 Pigtail (test report). Shanghai: Shanghai Testing \& Inspection Institute for Electrical Equipment (STIEE).

[32] Jiang, L., Wu, Z.Y., Zhang, X., Liu, H.M., Wang, Y. (2017). Measurement for cable shielding effectiveness of new energy vehicles. Safety and EMC, (5), 41-43.

Received May 29, 2019. Accepted August 30, 2019. 